\title{
Towards a Converged Network: Video Streaming over WiMAX vs. HSDPA in the Rural Environment
}

\author{
Odhiambo M.O., Amimo-Rayolla P. O. and Kurien A.M.
}

\begin{abstract}
- as the telecommunications industry evolves towards a converged IP-Core network the role of cellular/wireless broadband technologies in achieving Long Term Evolution (LTE) must be greatly evaluated. This would in effect provide lessons for improvement and blueprints for final adoption in NGN (Next Generation Networks). The emergence of BWA (broadband wireless access) as a reliable player, in some cases a substitute for fibre/ADSL, maybe attributed to poor or non-existent copper/fibre, largely inefficient telecommunications management and/or ineffective telecommunications policies in mostly underserviced areas. In this paper, video streaming over existing 3G high-speed packet networks is evaluated against WiMAX as alternatives to fibre/ADSL. Simulation results show WiMAX was found to be a viable and better alternative barring capital expenditure (CAPEX) and operational expenditure (OPEX) considerations. HSDPA was found to achieve rates per user equipment (UE) that meet QoS needs for codec streaming Q-levels of 16 and $64 \mathrm{kbps}$. The $16 \mathrm{kbps}$ codec rarely requires extensive preroll buffer requirements. However, WiMAX was found to perform better by at least three times for throughput and capacity.
\end{abstract}

Index Terms - H.263/MPEG-4, HSDPA, UMTS, WiMAX

\section{INTRODUCTION}

$\mathrm{T}$ he deployment of 3rd Generation (3G) mobile networks has seen an increase in a variety of cellular services including IP-based services such as data, mobile-TV and video streaming. This variety in service provisioning has been boosted more by user mobility enhancing location-based services. The consumption of these services is however, still largely dependent on their type, nature and on consumer willingness to pay for a service. For instance, video and VoIP services are most likely exploited for person-to-person communication with businesses settling for high-speed internet. While the broadband market, and indeed, most of the telecommunications market is driven by subscriber preferences and needs, the access technology defines the limits of the application and service menu offered by the operators and service providers.

Manuscript submitted March 31, 2009. Dr. Marcel Odhiambo, is the HoD Department of Electrical and Mining, UNISA, Florida Campus, Johannesburg. (OhangMO@unisa.ac.za); Otieno Amimo-Rayolla is with the French South African Technical Institute in Electronics (F'SATIE), Pretoria, South Africa (Amimo.Rayolla@,fsatie.ac.za) and Anish Kurien, is a senior lecturer with the Department of Electrical and Electronics, Tshwane University of Technology, Pretoria, South Africa. (KurienAM@tut.ac.za)
High-Speed Downlink Packet Access (HSDPA) is an extension of the Universal Mobile Telecommunications System (UMTS) that enhances the downlink by increasing the downlink throughput by about 5 times that of Wideband Code Division Multiple Access (WCDMA) up to a theoretical maximum of $14 \mathrm{Mbps}$ [15]. This is done by introducing fast node-B scheduling, Hybrid-ARQ, adaptive modulation schemes (AMC), and fixed spreading factor while doing away with fast power control, variable spreading factor and soft handoff. HSDPA has the advantage of having already existing Global Systems for Mobile communications (GSM) networks that in some cases may only need software or hardware upgrades, reducing overhead costs considerably [7]. Wireless Interoperability for Microwave Access (WiMAX) on the other hand introduces Orthogonal Frequency Division Multiplexing/Multiple Access (OFDM/A) and Non-Line Line Of Sight (NLOS) implementations enabling the delivery of last mile wireless broadband access as an alternative to cable and Asymmetric Digital Subscriber Line (ADSL). This ensures wider reach, better adaptability to channel conditions and an overall performance of symmetric broadband speeds, with a theoretical maxima of up to 5 times that of HSDPA (70Mbps) $[20,21,22]$. In spite of such enormous advantages for WiMAX, the lack of any widely deployed networks especially in the rural environment makes the prospect for exploiting such potential a difficult idea. The Capital Expenditure (CAPEX) of rolling out a WiMAX network could be large to make it impossible to successfully launch a viable business model.

It is broadly accepted in industry that access technologies can only support, with a satisfactory user's Quality of Service (QoS) perception, a limited set of services without incurring major spectral efficiency costs to a system $[19,94]$. A number of studies, such as $[3,6,12,13,14]$, have evaluated QoS parameters of video streaming services over 3G/HSDPA and Wi-LAN/WiMAX. These studies have, with a good degree of satisfaction proved QoS parameters are met with acceptable Peak Signal-to-Noise Ratio/Mean Opinion Score (PSNR/MOS) and percentage-loss indices adequately met under 'standards' (3GPP and WiMAX Forum) QoS class requirements. This is particularly important for trace based simulations like the one carried out in this paper. The PSNR measures the quality of reconstruction of lossless compression codecs as a ratio of the original video signal power, and the noise signal power introduced by compression. PSNR typical values under wired conditions are 30-50dB. However, for the wireless environment, quality loss values of $20-25 \mathrm{~dB}$ and greater are acceptable. 


\section{High-SPEed Downlink PACKet AcCess - HSDPA}

\section{Background}

High-Speed Downlink Packet Access (HSDPA) is an evolution of the W-CDMA standard Rel'5, designed to increase the available data rate by at least a factor of 5 or more. HSDPA supports services that generate large traffic loads such as interactive, background, streaming as well as conversational services in the downlink [2]. HSDPA therefore defines a new W-CDMA channel; the High-Speed Downlink Shared Channel (HS-DSCH) that supports higher capacity, reduced delay and higher peak data rate, of up to theoretical maximums of $14 \mathrm{Mbps}$ by multiplexing a large amount of power and code resources to a single user at and for a certain Transmission Time Interval (TTI). The HS-DSCH thus provides significant trunking benefits over Downlink Channel (DCH) for bursty high data rate traffic. HSDPA thus tightly couples features of Adaptive Modulation and Coding, fast Physical Layer Hybrid-ARQ and fast Packet Scheduling, and permit a per-TTI adaptation of the transmission parameters to the instantaneous variations of the radio channel quality to achieve a superior data rate $[9,10,15]$.

\section{Technology}

HSDPA specifies the HS-DSCH channel that replaces three fundamental features of other WCDMA channels - the variable spreading factor (SF), soft handoff and fast power control and instead uses:

- Adaptive Modulation and Coding (AMC).

- Fast packet scheduling at the Node B.

- Fast retransmissions from Node B (known as HARQ-Hybrid Automatic Repeat Request) to deliver the improved downlink performance.

- Shorter Transmission Time Interval (TTI)

- Multiple code operation

HARQ uses the concept of SAW (Stop And Wait) transmission protocol with soft combining. Chase Combining and Incremental Redundancy, where retransmissions contain different codings of the user data relative to the original transmission, are the two types of HARQ used in HSDPA. When a UE receives a corrupted packet, it saves it and combines it with subsequent retransmissions to formulate an error-free packet [7].

HSDPA has a logical channel structure and the main data bearer, HS-DSCH, is thus mapped onto a pool of physical channels (channelization codes) called HS-PDSCHs (High Speed Physical Downlink Shared Channel) to be multiplexed in time amongst the HSDPA users. Two new physical channels are also specified in HSDPA; the High Speed Shared Control Channel (HS-SCCH), a signalling carrier that informs the users when they are to be served as well as the necessary information for the decoding process and a reverse channel, the High Speed Dedicated Physical Channel (HS-DPCH), which carries ACK information and the Channel Quality Indicator (CQI) reports. The HS-DPCH consists of two parts, the first part and one slot length, carries the ACK for the HARQ process and the second part, two slots length and contains the CQI value. To aid the power control operation of the HS-DPCCH, an associated Dedicated Physical Channel (DPCH) is run for every user.

\section{WIRELESS INTEROPERABILITY FOR MICROWAVE ACCESS}

WiMAX, also known as the IEEE WirelessMAN air interface, is an emerging suite of standards for fixed, portable and mobile Broadband Wireless Access (BWA) in Metropolitan Area Networks (MAN). Though originally intended as a Broadband Fixed Wireless Access (BFWA) system serving as backhaul in a PMP (point-to-multi-point) network architecture, it has undergone modifications so that it can provide fixed, nomadic, portable and mobile wireless broadband connectivity without the need for direct line-of-sight with a base station. In a typical cell radius deployment of 3 to $10 \mathrm{~km}$, WiMAX Forum Certified systems can be expected to deliver capacity of up to $70 \mathrm{Mbps}$ per channel for fixed and portable access applications [20].

802.16 was originally designed for LOS conditions to operate in the frequency range of 10-66 GHz. Modifications to this specification have come in the form of support for frequencies between 2-11 GHz and different physical layers. Among these changes is the 256-carrier OFDM physical layer which enables better NLOS performance compared to single-carrier and CDMA. This has been enhanced in 802.16e standard OFDMA, with dynamic sub-channelization. The MAC-layer is built to support IP, ATM and Ethernet traffic through its convergence layer, with five levels of QoS at MAC level in the form of constant bit rate grant (UGS), real time polling (rtPS), enhanced real-time polling (ertPS), non-real-time polling (nrtPS), and best effort (BE) $[4,17]$.

Support for dynamic resource allocation for uplink and downlink both in TDD and full/half FDD mode is provided for in the MAC standard. The MAC PDUs (Protocol Data Units) can be of variable length, with support for concatenation and fragmentation, which is also available to the internal MAC-SDU (Service Data Unit). Concatenation and fragmentation ensure QoS parameters are effectively met by saving on overhead in headers through packet resizing. Package Header Suppression (PHS) helps in discarding redundant header information while terminals use a request/grant scheme to request for QoS according to their needs. Unlike in 802.11 where traffic is contention based, 802.16 data traffic over the MAC-layer is scheduled with the option of transmission over contention periods for certain types of bandwidth requests and ranging. This enhances flexibility and reduces latency. The MAC-layer also has a security sublayer performing encryption, authentication and encryption key exchange [4].

\section{STREAMING H.263/MPEG-4 OVER WIRELESS ACCESS}

Wireless streaming refers to content streamed over a wireless network to a packet switched data enabled terminal. A standardized solution allows a cost-efficient terminal implementation and enables interoperability between servers, networks, and terminals from different vendors. For instance all major mobile telecommunication equipment providers as well 
as streaming equipment providers support the 3GPP PSS (Packet Switched Service) standard. Wireless packet services have adopted available Internet technologies like the Real-Time Streaming Protocol (RTSP) and the Session Description Protocol (SDP) for session setup and control, and the Real-Time Transfer Protocol (RTP) for transporting real-time media such as video, speech, and audio $[8,5,18,19]$

The simulations used H.263 version $2(+)$ which can be transcoded to MPEG-4. Video transcoding refers to the conversion amongst video codecs e.g. H.263 to MPEG-4 and vice versa. Bit rate, frame rate and frame size may be changed. The H.263 standard combines the features of MPEG and H.261 [1] for very low bit rate coding making it easier to transport bursty channels. H.263 allows for the use of five standardized picture formats. These are the CIF (Common Intermediate Format), QCIF (Quarter-CIF), sub-QCIF, 4CIF and 16CIF. Picture formats are defined in terms of the number of pixels per line, the number of lines per picture and the pixel aspect ratio. The luminance component of the picture is sampled at these frame resolutions, while the chrominance components, $\mathrm{Cb}$ and $\mathrm{Cr}$, are downsampled by two in both the horizontal and vertical directions. Each picture in the input video sequence is divided into macroblocks, consisting of four luminance blocks of 8 pixels $\mathrm{x} 8$ lines followed by one $\mathrm{Cb}$ block and one $\mathrm{Cr}$ block, each consisting of 8 pixels $x 8$ lines.

\section{SimUlations}

To simulate video traffic over broadband wireless networks, a suitable streaming model must be formulated. Often, streaming servers emit traffic with infinitesimal silence periods in between the transmission blocks. There also exists significant correlation between and amongst the data units in streamed traffic. Usually, traffic on the air interface is likely to experience self-similarity effect since in all likelihood it remains bursty on diverse time scales. It is thus necessary to come up with a model that ensures the following:

1. The client receives, fairly, the same quality video under changing network conditions

2. One or a few clients do not utilize the entire bandwidth at the expense of other network users and

3. The streamed video is compatible with client equipment for viewing.

The streaming traffic was modelled as a Constant Bit Rate (CBR) source with the bit rate with Q-levels specified at $16 \mathrm{kbps}$ or $64 \mathrm{kbps}$.

\section{NS2 Set-up}

To evaluate the performance of H.263/MPEG-4 video streaming over HSDPA/WiMAX, a number of extensions or patches were made to the NS-2 simulator. In the $3 \mathrm{G}$ simulation, instances of UMTS nodes such as UE, Node B and RNC are instantiated in the extensions under the SEARCON project EURANE (Enhanced UMTS Radio Access Network Extensions) [16]. For the WiMAX simulation, the NIST (National Institute of Standards and Technology) extension was used with the 802.16 MAC extension instantiated. The network models, based on the system architectures are illustrated in Fig. 1 and 2. The UE/SS plays the role of a streaming client and a fixed host within the core network or in the Internet is the streaming server. It is important to implement algorithms that represent the family of transport protocols used in video streaming; UDP, RTP and RTCP

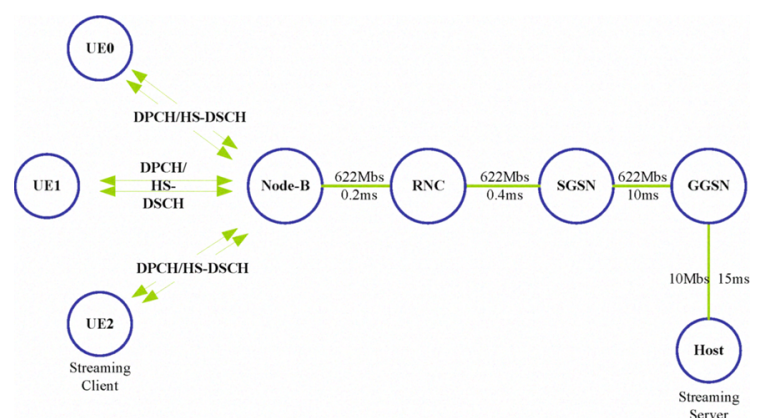

Figure 2: UMTS/HSDPA Simulation Network Model

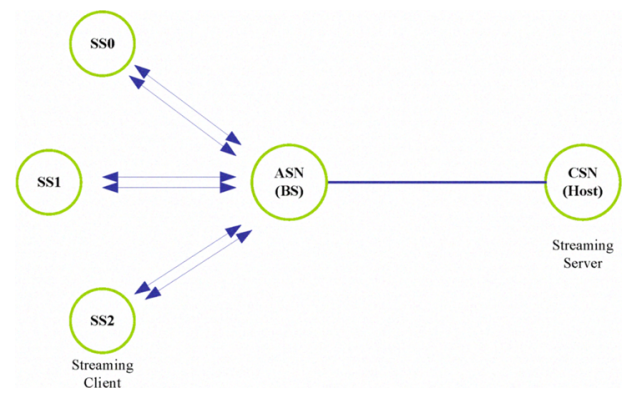

Figure 1: WiMAX Simulation Network Model

Unacknowledged mode (UM) was assumed; no interaction between the video client and server was and thus RTCP was not modelled. Likewise, RTP was also not modelled explicitly. However, all functions for packetization, packet sequence numbering and ordered delivery were modelled as UDP functions (myUDP) [11] in NS-2. The fundamental intention was to evaluate H.263/MPEG-4 video streaming over the radio interface. It was thus assumed that no packet losses, errors or congestion occur on either the Internet or the UMTS/WiMAX core network. Transfer delay over the Internet and the CNs was also assumed to be constant throughout the entire video streaming duration

\section{NS2 Simulation Model}

The NS2 simulation framework is as shown in Fig. 3 below. The coded videos (H.263/MPEG-4) are fed directly to the source transmitter while their empirical traces (network trace) are taken through a source agent (myTrafficTrace) to extract metric statistics before transmission. The result of the source UDP agents are sender trace files. At the receiver/sink the UDP sink agent extracts statistics for the receiver trace files. In the end there are 3 trace files; the sender trace file, the receiver trace file and the overall network trace file. These three files are then evaluated (post-processing) to extract the network performance metrics for throughput, delay, loss rate and jitter. The overall network trace has all the information that includes what is in the sender and receiver trace files. 


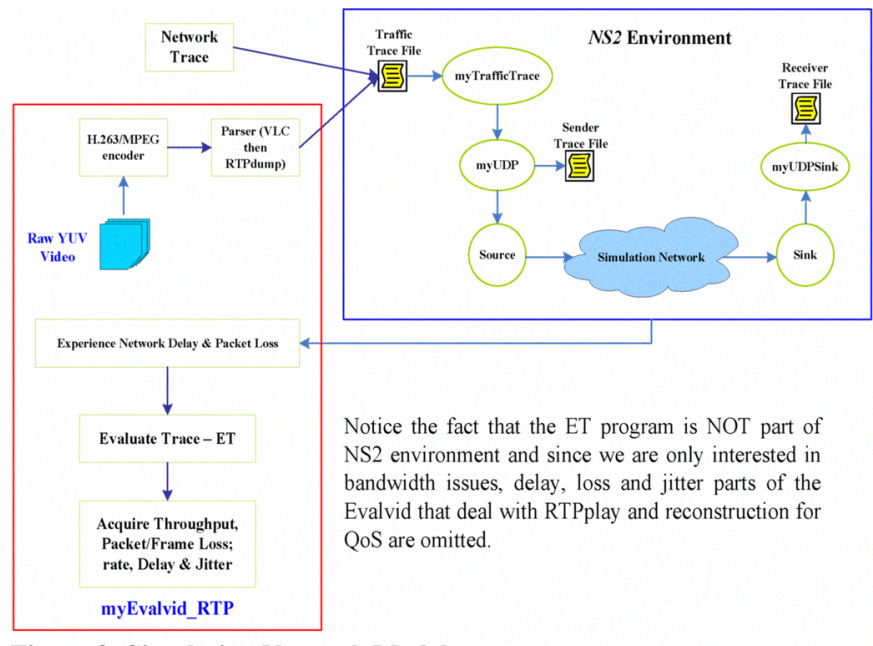

Figure 3: Simulation Network Model

\section{METRICS}

A set of measurements or deliverables that would assist in distinguishing the performance disparities between these two technologies were defined into mathematical formulae, coded in Perl and C, and used to parse the metrics from the trace files. These metrics included average rate, average delay and end-to-end delay

\section{Average Rate}

Consider $\gamma_{i}[n]$ as per TTI user throughput of user $i$ then:

$$
\gamma_{i}[n]=\frac{b_{i}[n]}{T T I} ; n=n_{1}, \ldots, n_{2}
$$

Where $b$ is the number of data bits delivered correctly to a user by PHY over a certain TTI, $n$, during the connection lifetime $n_{1}, n_{2}$. The instantaneous throughput of user $i$ is thus:

$$
\gamma_{i}=\frac{1}{n_{1}-n_{2}+1} \sum_{n_{1}}^{n_{2}} \gamma_{i}[m]
$$

$\gamma_{i}$ is a random variable that depends on path loss experienced by the user during the interval $n_{1}, n_{2}$ and intra-cellular interference.

If the statistical properties of all the users are the same, then the user throughput $R_{\gamma}$ follows a probability density function $f_{\gamma}$. The average user throughput is then defined as the statistical mean as follows:

$$
R_{\gamma}=\int_{-\infty}^{\infty} \gamma \cdot f_{\gamma} \cdot d \gamma
$$

\section{Delay and Jitter}

Assuming the streaming server and the client have synchronized clocks, the client can use the timestamp of the RTP packet and the local time that the packet is received in order to estimate the delay and the variation of the delay, frame jitter. Playout buffers absorb the jitter introduced by network delivery delays. The buffer can be as big as the entire video or as small as frame length. The "frame time" is determined by the time at which the last segment of a segmented frame is received. Below are mathematical equations for calculating delay and jitter either by frame or by packet time.

Consider $t_{P_{n}}$ as the receiver time stamp of the packet $n$ and inter-packet time ${ }^{{ }^{t}} P_{P_{0}}=0$, then Packet Delay is given by

$$
t_{P d_{n}}=t_{P_{n}}-t_{P_{n-1}}
$$

or Let $t_{F m}$ be the time-stamp of the last segment of the Frame $m$ and the inter-frame time ${ }^{i t} F_{0}=0$, The Frame Delay is then given by

$$
t_{F d_{m}}=t_{F_{m}}-t_{F_{m-1}}
$$

Jitter, however, is the variation in delay. If $\mathrm{N}$ is the number of packets, $M$ the number of frames and the average inter-packet time is given by $\overline{t_{N}}$, and the average inter-frame time given by ${ }^{{ }^{-}}{ }_{M}$ then

$$
\begin{aligned}
& \text { Packet Jitter, } j_{P}=\frac{1}{N} \sum_{i=1}^{N}\left(i t_{i}-i t_{N}\right)^{2} \\
& \text { and } j_{F}=\frac{1}{M} \sum_{i=1}^{M}\left(i t_{i}-i t_{M}\right)^{2}
\end{aligned}
$$

\section{RESUlTS AND DisCUSSION}

The effect of point-to-multi-point streaming was compared using 5-UE/SS with a random maximum speed of $3 \mathrm{kmph}$ for expected rates of $16 \mathrm{kbps}$ and $64 \mathrm{kbps}$. WiMAX was also evaluated for rates with 16QAM and 64QAM modulation schemes (MS) at a cyclic-prefix of $1 / 4$. $3 \mathrm{kmph}$ is the lowest achievable speed without compounding too much error in the considered Path Loss model. Practically, it is nearly standstill speeds and thus acceptable.

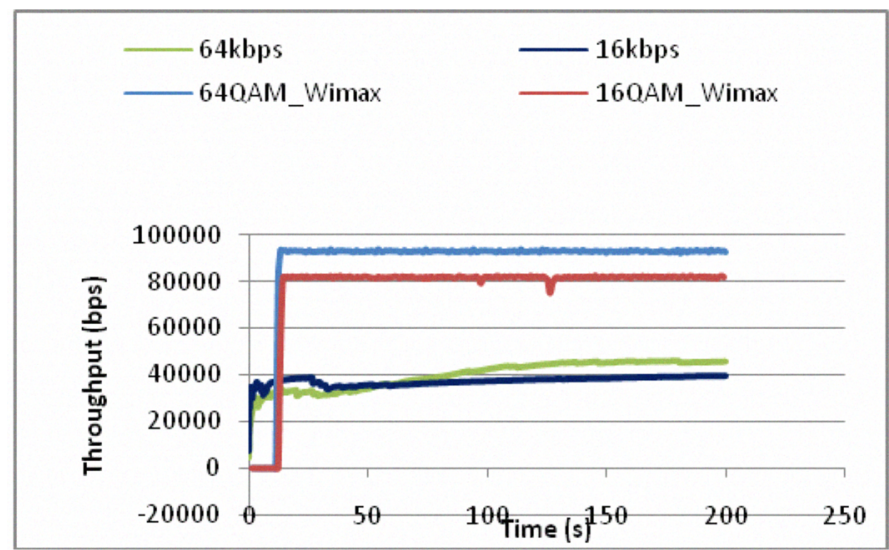

Figure 4: Average Throughput for 5-UE/SS 
The number of UE/SS was also increased from 5 to 12 , to 20 and finally from 20 to 50 . Other parameters tested included modulation and coding schemes and variation of Q-level between $16 \mathrm{kbps}$ to $64 \mathrm{kbps}$ expected rate. UEs were streamed with both the $16 \mathrm{kbps}$ and $64 \mathrm{kbps}$ video streams the SS were streamed with $64 \mathrm{kbps}$ expected rate only.

From Fig. 4, it can be see that the HSDPA curves oscillate initially while the WiMAX graph runs at zero for some time. This is attributed to call admission difficulty for HSDPA and packet collision during initial grant request for WiMAX. After the initial turbulence, the HSDPA rate for $16 \mathrm{kbps}$ averages at about $40 \mathrm{kbps}$ while for $64 \mathrm{kbps}$ it averages at $46 \mathrm{kbps}$ and remains steady for the period of the simulation. The WiMAX rate on the other hand averages at about $92 \mathrm{kbps}$ for a $64 \mathrm{kbps}$ expected rate with 64QAM MS used and 82kbps with 16QAM MS used for the same 6 nodes. The HSDPA traffic at $64 \mathrm{kbps}$, with good design, would require less than $1 \mathrm{sec}$ of playout buffer to ensure QoS at the client.

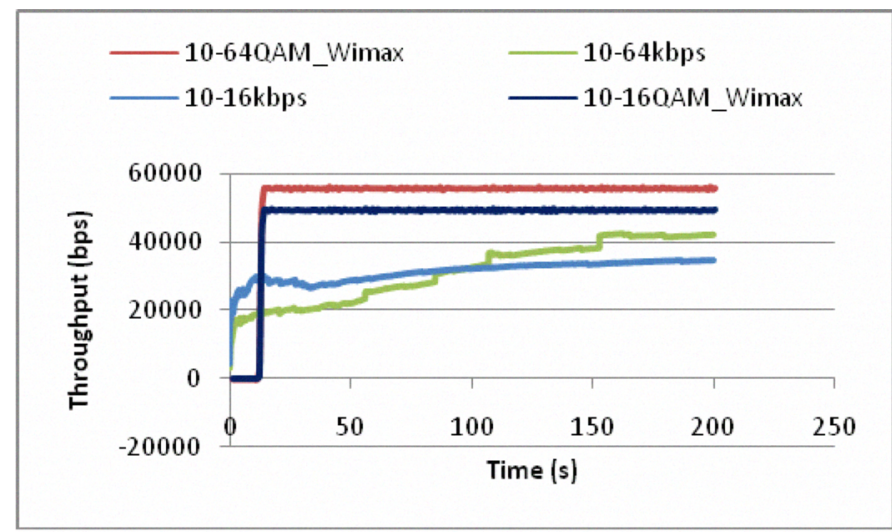

Figure 5: Average Throughput for $10 \mathrm{UE} / \mathrm{SS}$

From Fig. 5, it can be observed that the average rate per node reduces for all scenarios; Q-level, access technology or modulation scheme. Still, WiMAX had better performance with the exception of the streaming rate dropping below the expected rate of $64 \mathrm{kbps}$ for the first time streaming at $49 \mathrm{kbps}$ and $56 \mathrm{kbps}$ for 16QAM and 16QAM MS respectively. A minimum 2 second lead time buffer would thus be required to achieve optimal playout at the receiver. For HSDPA, the $16 \mathrm{kbps}$ video still streamed above the expected rate, but the $64 \mathrm{kbps}$ video streamed at below half of expected rate up to the first 100 seconds before it improved above $32 \mathrm{kbps}$. If the rate before 100 seconds is assumed to include all UEs, then a better design of buffering should be taken into account to ensure improved rate or optimal playout or a reduced number of UEs used.

Fig. 6 indicates that the delay experienced by the $64 \mathrm{kbps}$ HSDPA stream was much higher (up to about $38 \mathrm{~s}$ ) than that experienced by $16 \mathrm{kbps}$ stream (less than $8 \mathrm{~s}$ ). However, both were still at higher values than the $64 \mathrm{kbps}$ WiMAX stream (64QAM), but this is expected for an end-to-end delay with that kind of codec Q-level and access network. What is interesting to note is that while the end-to-end delay values increased, the average streaming rate remained relative stable implying uninterrupted pre-roll at the client. It is therefore safe to conclude that this delay only affects the queue at the pre-roll buffer.

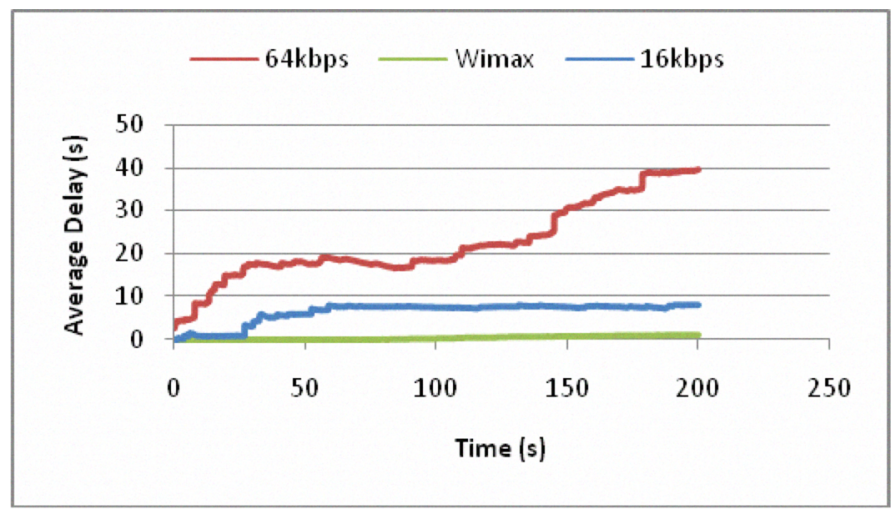

Figure 6: End-to-end delay for 5-UE/SS

This conclusion is further reinforced by an analysis of the average delay experienced by the nodes/clients. When the trace files are parsed for delay, very small values are obtained for average delay. For WiMAX (64QAM), $0.708 \mathrm{~s}$ was obtained, for HSDPA, 5.4325s and $2.0423 \mathrm{~s}$ was the average delay obtained for $64 \mathrm{kbps}$ and $16 \mathrm{kbps}$ respectively.

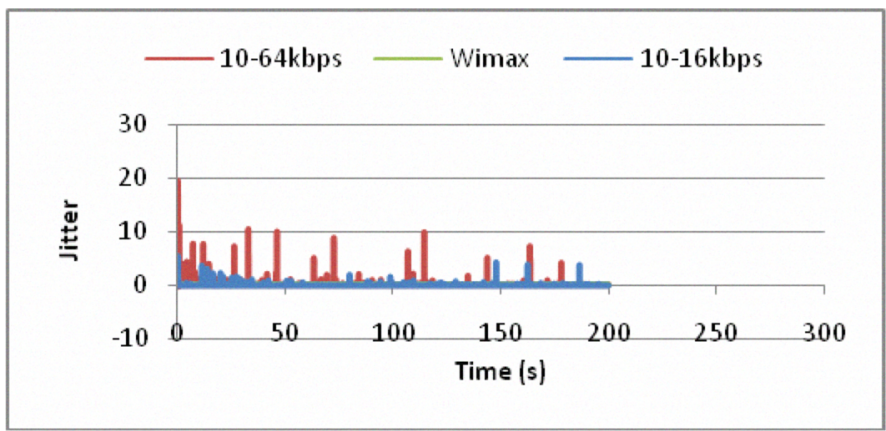

Figure 7: Jitter for 10-nodes

The jitter values for 5UE/SS were relatively low. For the WiMAX stream, the jitter values are uniform about the $\mathrm{x}$-axis at \pm 0.02 with the exception at the beginning when collision due to grant request caused sharp spikes. This value, as in the case of the HSDPA at $16 \mathrm{kbps}$, remains virtually zero. From Fig. 6 , the $64 \mathrm{kbps}$ stream records the highest spikes while the $16 \mathrm{kbps}$ and the WiMAX streams showed relatively low jitter except for the beginning when call admission is a problem.

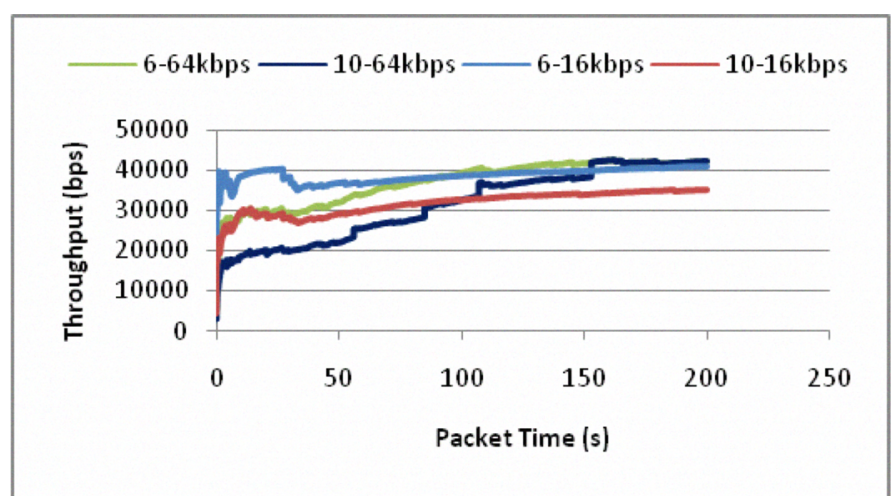

Figure 8: HSDPA rate comparison 
Fig. 7 and 8 show possible loading comparisons for HSDPA and WiMAX.

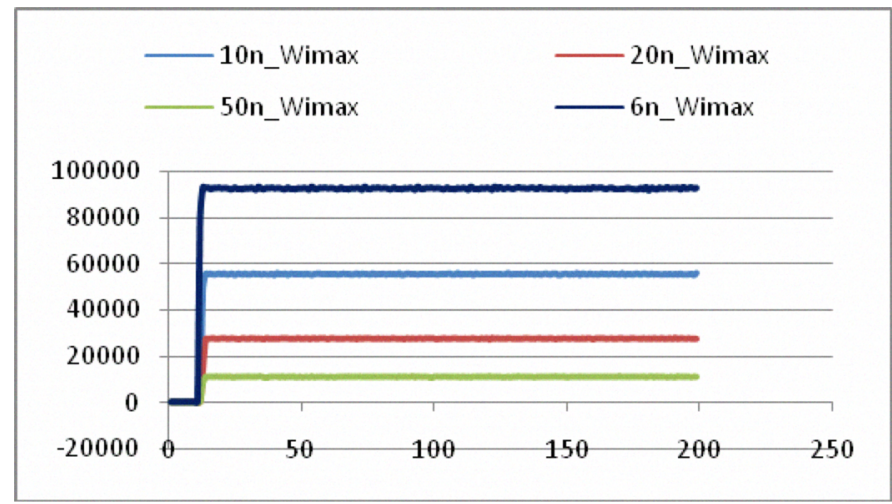

Figure 9: WiMAX rate comparison

\section{CONCLUSION}

Taking the ITU benchmark of $16 \mathrm{kbps}$ and PSNR of $20 \mathrm{~dB}+$, both HSDPA and WiMAX performed well on all of the Q-levels and increased cell-loading with the exception of increased loading above 10 UEs for HSDPA and 20 nodes for WiMAX. However, if this minimum rate is used as benchmark, playout buffering would be crucial to this success which in turn would impose extra costs on equipment or hardware size. It should be noted that at optimum loading an average minimum of 32kbps for both access technologies and for both Q-level streams are possible. This in effect means that a pre-roll buffer with at least $2 \mathrm{~s}$ for $64 \mathrm{kbps}$ and none for $16 \mathrm{kbps}$ would be needed.

It was observed that $16 \mathrm{kbps}$ video streams would be ideal for QoS rates. However, the danger lies in the visual quality that is only achievable if a higher quality bit-stream is used. A balance has thus to be struck between the better quality video (64kbps) and a better stream video (16kbps). What this would translate to in practical terms is two-fold.

1. A compromise between the two streams at $28 \mathrm{kbps}$ or $32 \mathrm{kbps}$ expected rates (static) or

2. On the fly encoding of video traffic to achieve rate-adaptation to the channel conditions.

Both these solutions would ensure a much better quality video at a rate that meets $\mathrm{QoS}$ and better cell-loading which was observed to be better for WiMAX as expected

\section{REFERENCES}

[1] 3GPP TS 25.435 v6.3.0, "UTRAN Iub Interface User Plane Protocols for Common Transport Channel data streams (Release 6)"

[2] 3GPP. Technical Specification Group Radio Access Network. High Speed Downlink Packet Access; Overall UTRAN Description. (3GPP TR 25.855 version 5.5.0).

[3] Alexiou A., Bouras C. and Igglesis V., "Multimedia Transmission over Third Generation Cellular Networks,"
[4] Alvarion, "Understanding the Radio Technologies of Mobile WiMAX, and their effect on network deployment optimization." White Paper 2006.

[5] Etoh M. and Yoshimura T., "Advances in Wireless Video Delivery," Proceedings of the IEEE, vol. 93, no. 1, January 2005

[6] Fitzek F. H. P. and Reisslein M., "MPEG-4 and H.263 Video Traces for Network Performance Evaluation", IEEE Network, 15, 2001.

[7] Forkel I., Klenner H. and Kemper A., "High Speed Downlink Packet Access (HSDPA) - Enhanced Data Rates for UMTS Evolution." P3 Solutions GmbH, RWTH Aachen University, Aachen, Germany,4th June 2005

[8] Gunnarsson F. and Bark G., "Providing Quality of Service for Streaming Applications in Evolved 3G Networks," Linkoping University, 29 January, 2004

[9] Holma H., and Toskala A., "WCDMA for UMTS," Wiley \& Sons, 2004.

[10]Holma H., Toskala A., "HSDPA/HSUPA for UMTS", John Wiley and Sons, 2006.

[11]Ke C. H., Yu C. Y., Chen R. S., Shieh C. K. and N. Chilamkurti, "MyEvalvid_RTP: a Evaluation Framework for More Realistic Simulations of Multimedia Transmission", International Journal of Software Engineering and Its Applications, vol. 2, no. 2, pp.21-32, 2008

[12]Ke C. H., C. K. Shieh, W. S. Hwang, A. Ziviani, "An Evaluation Framework for More Realistic Simulations of MPEG Video Transmission", Journal of Information Science and Engineering, vol. 24, no. 2, pp. 425-440, March 2008 (SCI)

[13]Lo A., Heijenk G. and Niemegeers I., "Performance Evaluation of MPEG-4 Video Streaming over UMTS Networks using an Integrated Tool Environment." Delft University of Technology, Netherlands, 2006

[14]Pliakas T., Kormentzas G, Skianis C. and Kourtis A., "Scalable Video Streaming Traffic Delivery in IP/UMTS Networking Environments," Journal of Multimedia, vol. 2, no. 2, April 2007.

[15]Qualcomm, "HSDPA for Improved Downlink Data Transfer," White Paper, Oct., 2004.

[16] SEACORN, End-to-end network model for Enhanced UMTS, Deliverable D3.2v2, 2003-10.

[17]SR Telecom, "WiMAX Technology: LOS and NLOS Environments," White Paper April 2004.

[18] Vukadinović V. and Karlsson G., "Resource Allocation for Wireless Video Streaming: Bit-Rate Adaptation and Packet Scheduling." KTH LCN Technical Report, Stockholm, Sweden, May 2006

[19] Vukadinović V. and Karlsson G., "Video Streaming in 3.5G: On Throughput-Delay Performance of Proportional Fair Scheduling," KTH LCN Technical Report, Stockholm, Sweden, 2006

[20] WiMAX Forum, "Mobile WiMAX - Part I: A Technical Overview and Performance Evaluation," August 2006

[21] WiMAX Forum, "Mobile WiMAX-Part II: A Competitive Analysis," February 2006.

[22] WiMAX Forum, "WiMAX and IMT-2000," White Paper, Jan., 2007. 